\title{
In-hospital cost comparison between the standard lateral and supercapsular percutaneously-assisted total hip surgical techniques for total hip replacement
}

\author{
Wade Gofton $^{1}$ - David A. Fitch ${ }^{2}$
}

Received: 22 April 2015 / Accepted: 1 May 2015 / Published online: 9 July 2015

(C) The Author(s) 2015. This article is published with open access at Springerlink.com

\begin{abstract}
Purpose The purpose of this study was to compare the inhospital costs associated with the tissue-sparing supercapsular percutaneously-assisted total hip (SuperPath) and traditional Lateral surgical techniques for total hip replacement (THR). Methods Between April 2013 and January 2014, in-hospital costs were reviewed for all THRs performed using the SuperPath technique by a single surgeon and all THRs performed using the Lateral technique by another surgeon at the same institution.

Results Overall, costs were $28.4 \%$ higher in the Lateral group. This was largely attributable to increased costs associated with transfusion $(+92.5 \%)$, patient rooms $(+60.4 \%)$, patient food $(+62.8 \%)$, narcotics $(+42.5 \%)$, physical therapy $(+52.5 \%)$, occupational therapy $(+88.6 \%)$, and social work $(+92.9 \%)$. The only costs noticeably increased for SuperPath were for imaging $(+105.9 \%)$, and this was because the SuperPath surgeon performed intraoperative radiographs on all patients while the Lateral surgeon did not.

Conclusions The use of the SuperPath technique resulted in in-hospital cost reductions of over $28 \%$, suggesting that this tissue-sparing surgical technique can be cost-effective primarily by facilitating early mobilisation and patient discharge even during a surgeon's initial experience with the approach.
\end{abstract}

David A. Fitch

david.fitch@ortho.microport.com

1 Ottawa Hospital - Civic Campus, J153-1053 Carling Ave., Ottawa, Ontario K1Y4E9, Canada

2 MicroPort Orthopedics Inc., 5677 Airline Rd., Arlington, TN 38002 , USA
Keywords Total hip replacement $\cdot$ Economic analysis . Supercapsular percutaneously-assisted total hip · SuperPath · Tissue-sparing $\cdot$ Lateral approach

\section{Introduction}

The economic burden of osteoarthritis $(\mathrm{OA})$ continues to grow as rising life-expectancy and obesity rates contribute to increased incidence of the condition. In 2008, 13.4 million patients in the United States aged 65 years or older incurred some expense due to treatment of OA resulting in health care expenditures of $\$ 24.8$ billion [1]. Total hip replacement (THR) is one of the most common treatments of OA in the hip joint, with annual volumes expected to reach 572,000 THRs in the United States alone by 2030 [2]. As a result, THR represents an excellent target for reducing the overall economic burden of OA.

One aspect of THR with the potential to reduce costs is the surgical technique. The supercapsular percutaneously-assisted total hip (SuperPath ${ }^{\circledR}$, MicroPort Orthopedics, Arlington, TN, USA) surgical technique is a tissue-sparing approach that preserves the external rotators and does not require the cutting of any muscles or tendons to access the capsule [3]. Recently published multicentre results for this technique showed substantial reductions in length of stay, 30-day readmission rates, and transfusion rates compared to the national average in the United States [4]. That study also provided examples of postdischarge cost savings associated with the reduction of these key variables. This is significant as post-discharge costs have been shown to account for nearly $40 \%$ of a THR episode of care [5].

While potential post-discharge savings have been shown, the in-hospital cost benefits associated with this technique have yet to be examined. The primary objective of this study 
was to compare the in-hospital costs associated with the SuperPath and standard Lateral surgical techniques. The standard Lateral, or Hardinge, technique was first described in 1982 [6], and has been used in approximately $40 \%$ of THRs captured in the National Joint Registry of England, Wales, and Northern Ireland since 2008 [7]. It is anticipated that inhospital costs will be reduced for the SuperPath technique when compared to the traditional Lateral due to the minimization of soft-tissue damage and ability of patients to ambulate faster.

\section{Methods}

In-hospital costs were reviewed for all SuperPath THRs performed by a single surgeon and all standard Lateral THRs performed by another surgeon at the same institution between April 2013 and January 2014. April 2013 was selected as the start date because this is when the SuperPath surgeon performed his first THR using the technique. The total costs, both direct and indirect, minus the cost of implants were considered in the analysis. Direct costs were defined as those attributed to providing direct patient care including salaries, supplies, and equipment amortisation. Indirect costs were defined as overhead allocations based on a percentage of the activity in the functional centre.

All aspects of cost associated with an in-hospital episode of care were considered including secondary items like patient food services. An episode of care was defined as beginning at the time of admission prior to the THR procedure and ending at the time of discharge from the hospital. Cost comparisons were presented as the percent difference between the two groups to protect proprietary hospital costing information. Cost per patient values were used instead of total costs to account for the different patient numbers in each group.

\section{SuperPath technique overview}

The SuperPath technique was performed as described by Chow et al. [3] The patient was positioned in the lateral position with the hip in $45^{\circ}$ of flexion and $10-15^{\circ}$ of internal rotation. A 6- to 8-cm incision initiated at the greater trochanter in line with the femoral axis, to the level of the gluteal fascia was made. The gluteal fascia was incised and the gluteus maximus was split in line with the fibres. The interval between the between the gluteus medius and minimus was identified. A blunt holeman was placed between the capsule and the minimus, followed by another blunt holeman between the capsule and the piriformis. The sciatic nerve remained protected by the intact short external rotators. The capsule was incised in line with the incision from the base of the greater trochanter to approximately $1 \mathrm{~cm}$ proximal to the acetabular rim. The capsule was elevated as a flap for $1 \mathrm{~cm}$ anterior and posterior to improve visualisation and the blunt holemans were placed intracapsular. The femur was reamed and broached without dislocation and with the femoral head intact to minimise the potential for femoral neck fracture and ensure restoration of the normal anatomic femoral version. Starting in the anterior portion of the piriformis fossa, the entry reamer was used to open the canal and a canal feeler was used to ensure the location. A calcar punch was used to open the femoral neck and head to allow passage of the broaches. Consecutive broaches were used until the broach was stable at a level relative to the greater trochanter as predetermined via templating. The superior aspect of the broach then served as a cutting template and the head was extracted. A bone hook in the implant and neutral rotation aided in anterior displacement of the femur as a trial cup was placed into the acetabulum. A portal placement guide allowed for the placement of a reaming cannula just posterior to the trochanter in line with the planned acetabular placement. The cannula was kept close to the femur to ensure that it was well away from the sciatic nerve. Reamer baskets were placed through the small incision and the small hex reaming shaft was passed through the cannula, allowing reaming with preservation of the short external rotators. The definitive cup and polyethylene liner were placed in a similar fashion with the option for alignment guides as desired. A trial head and neck were placed and, using a blunt trochar to push the femur with an assistant abducting or adducting the leg and rotating the femur as directed, the neck was reduced into the femoral head. The femur was assessed for stability and definitive components were selected. The trial components were separated and removed. The definitive femoral head was positioned and then a monoblock or modular femoral stem were placed and the reduction manoeuvre was repeated. The capsule was closed with a suture as was the gluteal fascia and skin.

\section{Lateral technique overview}

The direct lateral approach was a slight modification of that initially described by Hardinge [6]. Patients were positioned in the lateral position and an incision approximately $10-15 \mathrm{~cm}$ in length was centered over the greater trochanter. The gluteal fascia and iliotibial band were incised in line with the skin incision and a large retractor positioned. Cautery was used to elevate the inferior $50 \%$ of the gluteus medius tendon off of the greater trochanter, leaving a cuff of tendon to allow a suture repair of the tendon at closure. Distally, the incision passed down to bone through the vastus lateralis in line with the fibers. Proximally, the gluteus medius was split in line with the fibres to the level of the acetabulum. The gluteus minimus and capsule were split as a single layer in line with the medius. The ligament of bigelow was released to facilitate dislocation and determine the level of femoral neck osteotomy. Dislocation was achieved by full adduction and external rotation. At 
closure, the capsule and minimus were closed with a figure-ofeight vicryl suture. The gluteus medius was repaired in a similar fashion. Interrupted figure-of-eight vircyl was used to close the iliotibial band, followed by interrupted suture and staples for the skin layer.

\section{Results}

There were 49 SuperPath and 50 Lateral THRs performed during the selected time period. Table 1 shows the patient demographics for each group. The mean length of stay was 2.1 days (range, 1-4 days) for the SuperPath group and 5.1 days (range, 2-26) for the Lateral group. The mean total in-hospital cost per patient in the Lateral group was $28.4 \%$ higher than that for the SuperPath group. Table 2 shows a breakdown of in-hospital cost categories and which technique had higher costs for each. Imaging costs were those associated with obtaining and interpreting any imaging (e.g. radiographs, ultrasound, computed tomography) performed during the episode of care. Narcotics costs included those associated with the costs of the drugs, distribution and monitoring, pharmacy labour, and intravenous admixture. Laboratory testing costs included any related to laboratory testing (e.g. microbiology, routine chemistry, routine haematology, pathology) performed during the episode of care.

\section{Discussion}

The in-hospital costs for the SuperPath and standard Lateral approaches were compared to determine if use of a tissuesparing surgical technique resulted in any in-hospital cost benefits. It was anticipated that costs would be reduced for SuperPath patients, as the technique has several features that allow for early patient mobilisation and in turn reduced length of stay. SuperPath utilises the interval between the piriformis and the gluteus medius to access the hip capsule superiorly without requiring the cutting of muscles or tendons, which preserves the natural structures that resist dislocation. Positioning of the patient and femoral access are similar to that used when placing an intramedullary rod, and the femur is broached with the femoral head intact to theoretically reduce

Table 1 Patient demographics for the SuperPath and Lateral groups

\begin{tabular}{lll}
\hline & SuperPath & Lateral \\
\hline No. of patients & 49 & 50 \\
Male (\%)/female (\%) & $38 \% / 62 \%$ & $34 \% / 66 \%$ \\
Mean age (years) & 68.1 & 73.1 \\
Mean BMI & 29.4 & 30.1 \\
\hline
\end{tabular}

Table 2 In-hospital cost categories and comparison for the two groups

\begin{tabular}{ll}
\hline Cost Category & $\begin{array}{l}\text { Group with higher costs } \\
\text { (\% Higher) }\end{array}$ \\
\hline Overall & Lateral $+28.4 \%$ \\
Admissions & SuperPath $+1.9 \%$ \\
Operating room & SuperPath $+0.1 \%$ \\
Post-anaesthesia care unit & SuperPath $+13.5 \%$ \\
Transfusions & Lateral $+92.5 \%$ \\
Imaging & SuperPath $+105.9 \%$ \\
Narcotics & Lateral $+42.5 \%$ \\
Laboratory testing & Lateral $+17.0 \%$ \\
Patient room & Lateral $+60.4 \%$ \\
Patient food & Lateral $+62.8 \%$ \\
Physical therapy & Lateral $+52.5 \%$ \\
Occupational therapy & Lateral $+88.6 \%$ \\
Social work & Lateral $+92.9 \%$ \\
\hline
\end{tabular}

the risk of periprosthetic fracture during preparation. In contrast, the Lateral approach requires the dissection of a significant portion of the gluteus medius, and often the minimus, as well as splitting of the iliotibial band. This muscular dissection likely leads to increased postoperative pain requiring more narcotics usage, decreased postoperative abductor strength, and reduced overall function and mobility that may all contribute to increased in-hospital costs.

The results confirmed what was expected and showed that mean total costs per patient were $28.4 \%$ higher in the Lateral group. The Lateral group also had higher costs in nearly all individual cost categories, with imaging costs being the major exception. Mean imaging costs were $105.9 \%$ higher in the SuperPath group. This was largely attributable to radiograph costs, which were $198.8 \%$ higher for SuperPath. Although not required by the technique, the SuperPath surgeon collected radiographs on all patients as a precaution because he was performing these procedures during his learning curve with the technique. The Lateral surgeon did not routinely collect radiographs and therefore had substantially less cost associated with this activity. Many surgeons consider intraoperative radiographs to be standard of care. If the costs of routine radiographs were added to the Lateral group, then the mean overall costs per patient would increase to $29.7 \%$ higher for the Lateral group.

The Lateral group had at least $42 \%$ higher costs associated with patient rooms, patient food, physical therapy, narcotics, transfusions, occupational therapy, and social work. Several of these differences were attributable to the decreased length of stay observed in the SuperPath group. The average length of stay for the Lateral group (5.1 days) was over twice that of the SuperPath group (2.1 days), so it is not surprising that the costs associated with patient rooms (Lateral $+60.4 \%$ ) and food services (Lateral $+62.8 \%$ ) were over double for the 
Lateral group. Additionally, physical therapy costs were $52.5 \%$ higher in the Lateral group because, in general, physical therapists made daily visits to patients during their hospital stay. The finding of reduced length of stay agrees with the previously cited multicentre study that found the length of stay for SuperPath patients to be almost exactly half the national average in the United States (1.6 vs. 3.3 days) [4].

Other cost differences, such as for transfusions, were not directly attributable to reduced length of stay. Transfusion costs were $92.5 \%$ higher in the Lateral group. The institution had a routine anticoagulation protocol in place for all patients when the included THRs were performed. Under this protocol, patients with significant stroke risk (e.g. atrial fibrillation, stents) received tranexamic acid prior to surgery. There was also a routine transfusion protocol that covered all included THRs on the unit. Due to the identical anticoagulation and transfusion protocols, the reduction in transfusions can be at least partly attributed to the tissue-sparing nature of the SuperPath procedure. This finding also agrees with the described recent multicentre study showing a substantial reduction in transfusion rates for this technique when compared to the national average in the United States [4]. Transfusions have been associated with increased complications, such as infections $[8,9]$. This suggests that the reduction in transfusions seen in the SuperPath group may have the potential to decrease not only in-hospital costs but also those associated with treating complications related to transfusions.

Narcotics costs were $42.5 \%$ higher in the Lateral group. This included the costs of the narcotics themselves (41.6\% higher), distribution and monitoring (35.9\% higher), and intravenous admixture $(35.6 \%)$. While some of the cost reduction in the SuperPath group can be attributed to the reduced length of stay, the tissue-sparing nature of the technique likely also plays a role. The SuperPath technique is intramuscular, requiring no muscle release, preserves the external rotators, and minimises stretching of the gluteus medius. All these factors may play a role in reduced postoperative pain and, in turn, narcotics usage. Initial reports with this technique support this suggestion by showing patient pain was well controlled using only oral medications [3]. The SuperPath technique is a hybrid of the superior capsular (SuperCap ${ }^{\circledR}$, MicroPort Orthopedics) and percutaneously-assisted total hip (PATH ${ }^{\circledR}$, MicroPort Orthopedics) approaches [10,11]. Reports for both of these techniques agree with that previously reported for SuperPath and show patient pain can be controlled with only oral medications $[12,13]$.

The average per patient cost associated with in-hospital occupational therapy was $88.6 \%$ higher in the Lateral group. Occupational therapists generally spend time with patients instructing them on various activities of daily living (e.g. sitting, putting on clothes, performing simple household tasks) to prepare them for their discharge from the hospital. In our unit, the occupational therapists are requested to review patients when the physician or physiotherapists feel their mobility is sufficiently limited to warrant a consult to avoid delays in a safe discharge home. The large reduction in these costs is possibly associated with improved early ambulation and function in the SuperPath group and the reduced number of visits required due to the decreased length of stay.

Social work costs were $92.9 \%$ higher for the Lateral group. Social workers facilitate arrangements for postdischarge care and help patients with practical aspects of THR, such as labour laws for requesting time off from work. One factor likely associated with the higher social work costs is that four $(8.0 \%)$ Lateral patients were sent to short-term rehabilitation facilities, while no SuperPath patients were. Arrangements to send patients to these facilities are managed by the social work group at our institution.

The mean per patient costs associated with admissions, operating room, and the post-anesthesia care unit were similar for the two groups. This suggests that use of the SuperPath technique does not result in increased operating room usage or patient time in the post-anesthesia care unit when compared to the standard Lateral approach. Further, the SuperPath technique does not require the use of specialty surgical tables or other equipment, and as such does not require any additional capital expenses from the hospital. Another potential benefit of the technique is that it can be performed with only a single assistant, which could result in increased operating room efficiencies.

\section{Limitations}

The study is not a randomised comparison of patients implanted by the same surgeon. Efforts were made to minimise the bias introduced from this limitation by selecting patients implanted during the same time period at the same institution. This ensured patients were treated with identical anticoagulation and transfusion protocols, while also receiving pre- and postoperative care at the same facilities. Patients in the Lateral group were five years older on average and this has the potential to play a role in increased hospital length of stay. Another limitation was the different levels of experience the implanting surgeons had with the two surgical techniques. The SuperPath surgeon was completing his first 49 THRs using the technique, while the other surgeon had significant experience with the Lateral technique. Future work is needed to examine if there any longer term benefits for SuperPath, as a recent report for the direct anterior approach showed similar outcomes when compared to the lateral at midterm follow-up [14]. Future studies of interest could also determine if there are benefits for select patient populations (e.g. obese patients) as have been examined recently for other surgical techniques [15]. 


\section{Conclusions}

In conclusion, the use of the SuperPath surgical technique resulted in in-hospital cost reductions of over $28 \%$ when compared to the standard Lateral performed at the same institution. Pre-operative and operative costs were similar between the two groups, with the majority of savings occurring due to reductions in length of stay, narcotics, transfusions, physical therapy, occupational therapy, and social work costs. These outcomes suggest this tissue-sparing surgical technique can be cost effective primarily by facilitating early mobilisation and patient discharge even during a surgeon's initial experience with the approach.

Open Access This article is distributed under the terms of the Creative Commons Attribution 4.0 International License (http:// creativecommons.org/licenses/by/4.0/), which permits unrestricted use, distribution, and reproduction in any medium, provided you give appropriate credit to the original author(s) and the source, provide a link to the Creative Commons license, and indicate if changes were made.

\section{References}

1. Soni A, Roemer M (2011) Top five most costly conditions among the elderly, Age 65 and Older, 2008: Estimates for the U.S. Civilian Noninstitutionalized Adult Population. Stat Brief \#327. Rockville, MD

2. Kurtz S, Ong K, Lau E, Mowat F, Halpern M (2007) Projections of primary and revision hip and knee arthroplasty in the United States from 2005 to 2030. J Bone Joint Surg Am 89(4):780-785. doi:10. 2106/JBJS.F.00222

3. Chow J, Penenberg B, Murphy S (2011) Modified micro-superior percutaneously-assisted total hip: early experiences \& case reports. Curr Rev Musculoskelet Med 4(3):146-150. doi:10.1007/s12178011-9090-y

4. Gofton W, Chow J, Olsen KD, Fitch DA (2014) Thirty-day readmission rate and discharge status following total hip arthroplasty using the supercapsular percutaneously-assisted total hip surgical technique. Int Orthop. doi:10.1007/s00264-014-2587-4

5. Bosco JA 3rd, Karkenny AJ, Hutzler LH, Slover JD, Iorio R (2014) Cost burden of 30-day readmissions following Medicare total hip and knee arthroplasty. J Arthroplast 29(5):903-905. doi:10.1016/j. arth.2013.11.006

6. Hardinge K (1982) The direct lateral approach to the hip. JBone Joint Surg Br 64(1):17-19

7. 11th Annual Report of the National Joint Registry of England, Wales, and Northern Ireland (2014). www.njrreports.org.uk

8. Carroll K, Dowsey M, Choong P, Peel T (2014) Risk factors for superficial wound complications in hip and knee arthroplasty. Clin Microbiol Infect : Off Publ Eur Soc Clin Microbiol Infect Dis 20(2): 130-135. doi:10.1111/1469-0691.12209

9. Frisch NB, Wessell NM, Charters MA, Yu S, Jeffries JJ, Silverton CD (2014) Predictors and complications of blood transfusion in total hip and knee arthroplasty. J Arthroplast 29(9 Suppl):189 192. doi:10.1016/j.arth.2014.03.048

10. Murphy S (2004) Technique of tissue-preserving, minimallyinvasive total hip arthroplasty using a superior capsulotomy. Oper Tech Orthop 14(2):94-101

11. Penenberg BL, Bolling WS, Riley M (2008) Percutaneously assisted total hip arthroplasty (PATH): a preliminary report. J Bone Joint Surg Am 90(Suppl 4):209-220. doi:10.2106/JBJS.H. 00673

12. Penenberg B (2014) Soft tissue-sparing total hip arthroplasty: the direct posterior approach. Cedars Sinai Adv Orthop (Winter 2014): 2

13. Wellman SS, Murphy AC, Gulcynski D, Murphy SB (2011) Implementation of an accelerated mobilization protocol following primary total hip arthroplasty: impact on length of stay and disposition. Curr Rev Musculoskelet Med 4(3):84-90. doi:10.1007/ s12178-011-9091-x

14. Reichert JC, Volkmann MR, Koppmair M, Rackwitz L, Ludemann M, Rudert M, Noth U (2015) Comparative retrospective study of the direct anterior and transgluteal approaches for primary total hip arthroplasty. Int Orthop. doi:10.1007/s00264-015-2732-8

15. Dienstknecht T, Luring C, Tingart M, Grifka J, Sendtner E (2013) A minimally invasive approach for total hip arthroplasty does not diminish early post-operative outcome in obese patients: a prospective, randomised trial. Int Orthop 37(6):1013-1018. doi:10.1007/ s00264-013-1833-5 